\title{
Impacto da escolaridade materna e paterna na perceção da imagem corporal em acadêmicos de Educação Física
}

\author{
Impact of maternal and paternal schooling in the body image perception \\ of physical education university students
}

\author{
D.A.S. Silva, I.M.M. Pereira, A.C. Cabral de Oliveira
}

ARTIGO ORIGINAL | ORIGINAL ARTICLE

\begin{abstract}
Objetivou-se verificar o impacto da escolaridade materna e paterna na perceção da imagem corporal em acadêmicos de Educação Física. Participaram do estudo 217 acadêmicos, com média de 20.6 anos de idade (DP $=0.6)$, sendo $54.8 \%$ do sexo masculino. Coletaram-se informações sobre perceção da imagem corporal por meio da escala de silhuetas de Stunkard et al. (1983). O nível de escolaridade dos pais e dados sociodemográficos foram coletados por questionário autoadministrado. Empregaram-se a estatística descritiva e a regressão logística multinomial, adotando-se nível de significância de 5\%. Em relação à perceção da imagem corporal, $41 \%$ dos alunos estavam insatisfeitos por magreza e $28.1 \%$ por excesso de peso. As chances de insatisfação por excesso de peso foram de sete a oito vezes maiores nos acadêmicos com escolaridade materna superiores a quatro anos de escolaridade, independentemente de sexo, idade, situação conjugal, nível econômico e curso. Não houve associação entre escolaridade paterna e imagem corporal. Conclui-se que há necessidade de uma educação básica e superior de qualidade com assuntos vinculados à perceção corporal e hábitos saudáveis, pois muitos acadêmicos apresentaram insatisfação com a imagem corporal e tal probabilidade foi maior em estudantes filhos de mães com mais de cinco anos de escolaridade.
\end{abstract}

Palavras-chave: imagem corporal, atividade motora, estudantes, indicadores demográficos, educação física e treinamento

ABSTRACT

The objective of this study was to verify the impact of maternal and paternal schooling in the body image perception of Physical Education university students. Two hundred and seventeen students took part in this study, with mean $20.6(\mathrm{SD}=0.6$ ) years of age, $54.8 \%$ were male. Information on body image perception was through the range of profiles of Stunkard et al. (1983). Maternal and paternal schooling level and sociodemographic data were collected by self-administered questionnaire. The descriptive statistics and multinomial logistic regression were used. The level of significance was $5 \%$. Regarding body image perception $41 \%$ was dissatisfied due to thinness, and $28.1 \%$ dissatisfied due to overweight. The probability of overweight dissatisfaction was seven to eight times higher in students whose maternal school length was 4 years or more, independently of the gender, age, marital status, socioeconomic status and course degree. There was no association between paternal schooling and body image. We conclude that there is a necessity of good quality education for all the schooling levels, because many academics unsatisfied with body image and the probability of overweight dissatisfaction was higher in individuals whose maternal school was five years or more.

Keywords: body image, motor activity, students, demographic indicators, physical education and training

Submetido: 10.12.2010 | Aceite: 08.03.2011

Diego Augusto Santos Silva. Universidade Federal de Santa Catarina/Centro de Desportos/Programa de Pós Graduação em Educação Física. Florianópolis, Santa Catarina, Brasil.

Indianara Magalhães Marques Pereira. Universidade Federal de Sergipe/Centro de Ciências Biológicas e da Saúde/ Departamento de Educação Física. Aracaju, Sergipe, Brasil.

Antonio César Cabral de Oliveira. Universidade Federal de Sergipe/Centro de Ciências Biológicas e da Saúde/ Núcleo de Pesquisa em Aptidão Física e Olimpismo de Sergipe/Departamento de Educação Física. Aracaju, Sergipe, Brasil.

Endereço para correspondência: Diego Augusto Santos Silva, Avenida Gonçalo Rollemberg Leite, 1960, Condomínio Alphaville I, Edf. Gama, Apto 1201, Bairro Suíça, CEP: 49050370 Aracaju, Sergipe, Brasil

E-mail: diegoaugustoss@yahoo.com.br 
A imagem corporal está relacionada ao grau de precisão com que o tamanho do corpo é percebido e ao nível de satisfação ou rejeição corporal (Slade, 1994). O processo de construção da imagem corporal está associado às conceções determinantes da cultura e da sociedade e é influenciável pelas dinâmicas interações entre o ser humano e o meio em que vive (Alves, Pinto, Alves, Mota, \& Leirós, 2009).

Os padrões ocidentais de estética têm perpetuado o estereótipo da associação entre magreza e beleza para o sexo feminino (Muennig, Jia, Lee, \& Lubetkin, 2008) e um corpo maior e mais volumoso para o masculino (Cafri \& Thompson, 2004). A cultura do padrão de beleza do ocidente não é atingível para a maioria dos indivíduos, que se frustram em relação à perceção de sua imagem corporal e, muitas vezes, recorrem às dietas, à prática de exercício físico exagerado e ao uso de diuréticos, laxantes e esteroides anabolizantes (Alves et al., 2009).

A literatura tem evidenciado que, independentemente da faixa etária da população, a insatisfação com a imagem corporal tem relação com transtornos mentais, como a depressão (Pimenta, Sánchez-Villegas, BesRastrollo, López, \& Martínez-González, 2009), distúrbios alimentares como anorexia e bulimia (Yeh et al., 2009) e baixa autoestima (Abraham, 2003). Essas condições afetam a qualidade de vida e refletem diretamente no estado de saúde e nas relações pessoais do cotidiano.

Pesquisas revelaram que a insatisfação com a imagem corporal é mais prevalente no final da adolescência e início da fase adulta, período em que há o ingresso no ensino superior ( $\mathrm{Al}$ Sabbah et al., 2009; Niskar, Baron-Epel, Gartysandalon, \& Keinan-Boker, 2009). Nesse sentido, universitários parecem constituir um grupo de risco para esse desfecho. Todavia, acadêmicos de Educação Física possuem disciplinas durante a formação que tratam diretamente do corpo e da relação com o mundo, no qual o ser humano está inserido. Além disso, têm disciplinas que tratam dos conceitos de educação em saúde, promoção da saúde e corporeidade. Por essas razões, especula-se que esses estudantes sejam bem resolvidos em relação à autopercepção da imagem corporal.

O nível de escolaridade dos pais é entendido pela United Nations Educational, Scientific and Cultural Organization (UNESCO) como "capital cultural" dos pais para os filhos e tem sido considerado indicador socioeducacional por condicionar a chance de escolarização dos filhos e a própria ambiência cultural da família (Kappel, 2007). A educação tem o impacto de perpetuação do ciclo de pobreza, uma vez que pais com baixa escolaridade têm dificuldade em garantir maior nível de escolaridade para os filhos, de tal forma que se gera um ciclo vicioso da pobreza entre gerações (Lamy Filho, Medeiros, Lamy, \& Moreira, 2011). Por essa razão, o nível de escolarização dos pais está diretamente associado à renda familiar e pode refletir em todo processo de formação intelectual do jovem.

O grau de bem-estar e vulnerabilidade dos jovens na sociedade está intimamente relacionado com os recursos materiais e educacionais de suas famílias. A disponibilidade de recursos materiais, refletida em certa medida pela renda familiar, está fortemente associada a um conjunto de fatores que podem determinar a inserção social e as relações entre as pessoas. Famílias em que os pais apresentam baixa escolaridade têm dificuldade de acessar patrimónios culturais e não apresentam tanta facilidade de acessar e interagir com os meios de comunicação, pois tais recursos ainda são dispendiosos no Brasil (Kappel, 2007).

Tendo em vista que a construção da imagem corporal é determinada pela relação do ser humano com a cultura e sociedade em que vive, como destacado por Frost (2005) em que a aparência é parte inevitável da identidade em uma sociedade capitalista que estimula desejos de corpos perfeitos e de beleza perfeita. E também como evidenciado por Alves et al. (2009) em uma investigação de revisão da 
literatura em que explicitaram que, enquanto membros da cultura ocidental, os sujeitos são confrontados diariamente, por meio da mídia, com verdadeiros modelos estéticos, que impõem ou criam o desejo da procura de um enquadramento nesses padrões de beleza que sofrem alterações de acordo com a cultura e a época, acredita-se que há necessidade de investigar a perceção da imagem corporal de universitários de Educação Física.

Além disso, a escolaridade dos pais pode refletir nas relações e inserção social dos jovens, pois como destaca Kappel (2007), a escolaridade é uma proxy para a renda e quanto maior o nível de escolaridade maior o acesso à educação, saúde, cultura e lazer. Por isso, especula-se que a perceção da imagem corporal possa ser afetada pelo nível de instrução dos pais. No entanto, em consulta nas bases de dados (Pubmed, Web of Science, Scopus, Lilacs, Scielo), no período de janeiro de 2000 a agosto de 2010, com os termos "body image", "weight perception" e "body size" associados aos termos "educational status" "educational achievement", "maternal educational status" e suas respectivas traduções para o português, não se identificou nenhum estudo que relacionasse escolaridade dos pais com imagem corporal, o que impede que se conheça a magnitude dessa associação.

Assim, o presente estudo tem como objetivo verificar o impacto da escolaridade materna e paterna na perceção da imagem corporal em acadêmicos de Educação Física.

\section{MÉTODO}

O presente estudo faz parte do projeto de pesquisa "Avaliação da atividade física, estilo de vida e variáveis associadas de universitários", tendo sido aprovado por um Comitê de Ética em Pesquisa com Seres Humanos (CAAE - 0163.0.107.000-09).

\section{Amostra}

Este estudo descritivo, com delineamento transversal, teve como população alunos de ambos os sexos, que estavam matriculados no curso de Educação Física - licenciatura ( $n=$ 149) e bacharelado $(n=145)$, no segundo semestre de 2009 de uma universidade pública do estado de Sergipe, Brasil, localizada na cidade de São Cristóvão.

O presente estudo faz parte de um projeto com vários desfechos em saúde e, por isso, foram calculados vários tamanhos de amostra, considerando-se orientações para amostragem aleatória simples. $\mathrm{Na}$ atual pesquisa, considerou-se o cálculo de tamanho de amostra, por curso, adotando-se erro tolerável de $5 \%$, nível de confiança de $95 \%$, prevalência de insatisfação com a imagem corporal de $77 \%$ (Quadros, Gordia, Martins, Silva, Ferrari, \& Petroski, 2010) e acréscimo de 5\% para eventuais perdas e recusas. Dessa forma, a amostra foi estimada em 102 sujeitos do curso de licenciatura e cem de bacharelado.

Após o cálculo, que estimou a quantidade de alunos necessária em cada curso, foi realizado um sorteio pelo programa Microsoft Office Excel $^{\circledR}$ do Windows para escolha dos sujeitos. Os sorteados foram contatados em sala de aula a partir de uma lista de matriculados distribuída pela instituição.

Foram considerados elegíveis todos os alunos de ambos os cursos matriculados. Alunos sorteados que não foram encontrados nas disciplinas, que estavam matriculados por três vezes em diferentes semanas, foram considerados perda amostral. Os estudantes que não quiseram responder ao instrumento foram considerados recusa. Utilizou-se como critério de exclusão apresentar alguma condição física que impossibilitasse responder ao questionário autoadministrado.

\section{Instrumentos e Procedimentos}

As informações referentes à perceção da imagem corporal foram obtidas por autoavaliação, utilizando-se a escala de nove silhuetas corporais propostas por Stunkard et al. (1983) e empregada em estudos com universitários (Quadros et al., 2010). O conjunto de silhuetas foi mostrado aos universitários, seguido das perguntas "Qual silhueta melhor representa sua 
aparência física atual (real)?" e "Qual silhueta você gostaria de ter (ideal)?". Para verificar a insatisfação com a imagem corporal, utilizou-se a diferença entre a silhueta real e a ideal. Quando a diferença foi igual a zero, o indivíduo foi classificado como satisfeito; se diferente de zero, como insatisfeito. Quando a diferença foi positiva, o indivíduo foi considerado como insatisfeito por excesso de peso e, quando negativa, como insatisfeito por magreza.

A escolaridade materna e paterna foi avaliada pela pergunta "Até que série/ano, seu (sua) pai (mãe) completou na escola?”, categorizada conforme a divisão do ensino brasileiro: $0-4$, $5-8,9-11,12$ ou mais anos de estudo.

As variáveis que foram controladas nas análises entre imagem corporal e escolaridade materna e paterna foram as sociodemográficas - sexo (masculino ou feminino), idade $(\leq 20$ anos; $>20$ anos), situação conjugal (com companheiro; sem companheiro), curso (licenciatura; bacharelado), nível econômico -, coletadas por meio do instrumento da Associação Brasileira de Empresas de Pesquisa (2008), que divide a população brasileira em categorias, conforme ordem decrescente de poder de compra: "A", "B", "C", "D" e "E". No presente estudo, agruparam-se os níveis económicos "A" e "B" e os níveis "C", "D" e "E".

A coleta de dados ocorreu no segundo semestre de 2009, no Departamento de Educação Física da instituição, e contou com a participação de uma professora de Educação Física, que distribuiu o questionário autoadministrado aos alunos sorteados em uma sala de aula da instituição após a assinatura do Termo de Consentimento Livre e Esclarecido.

\section{Análise Estatística}

Empregou-se a estatística descritiva, com valores médios, desvio padrão, frequência absoluta e relativa e intervalos de confiança. Para analisar a associação entre imagem corporal e escolaridade materna e paterna, utilizou-se a regressão logística multinomial, pois o desfecho (imagem corporal) apresentara três categorias. A categoria de referência foi satisfação com a imagem corporal. Estimaramse odds ratio e intervalos de confiança, tanto na análise bruta quanto na ajustada pelas variáveis sociodemográficas (sexo, idade, situação conjugal, nível econômico e curso). Em todas as análises foi adotado nível de confiança de 95\%.

\section{RESULTADOS}

O presente estudo faz parte de um projeto de pesquisa com vários desfechos em saúde e, por isso, coletaram-se dados de 217 académicos, sendo 105 de licenciatura e 112 de bacharelado, com média de 20.6 anos de idade (DP $=0.6$ ). A variável imagem corporal não apresentou nenhuma resposta ignorada. Dentre as variáveis independentes, o nível econômico apresentou três respostas ignoradas.

Na Tabela 1, observa-se que a maior parte dos estudantes investigados foi do sexo masculino (54.8\%), com idade até 20 anos (59.5\%), que viviam sem companheiro (92.6\%), de nível econômico alto $(62.8 \%)$, do curso de bacharelado (51.6\%), com escolaridade materna e paterna entre nove e 11 anos completos (41.0 e $36.4 \%$, respetivamente). Em relação à perceção da imagem corporal, $41 \%$ estavam insatisfeitos por magreza e $28.1 \%$ insatisfeitos por excesso de peso.

Em relação à perceção da imagem corporal, 30.6\% (IC 95\%: 21.3-39.9) dos académicos do sexo feminino e $31.1 \%$ (IC 95\%: 22.7-39.5) do masculino estavam satisfeitos com a imagem corporal. Em relação à insatisfação por magreza, 34.7\% (IC 95\%: 25.1 - 44.3) do sexo feminino e $46.2 \%$ (IC 95\%: 37.1-55.3) do masculino relataram essa condição. Além disso, 34.7\% (IC 95\%: 25.1-44.3) dos universitários do sexo feminino e $22.7 \%$ (IC $95 \%$ : 15.1-30.3) do masculino estavam insatisfeitos por excesso de peso (Figura 1).

A Tabela 2 apresenta os resultados da análise de regressão multinomial da imagem corporal em relação à escolaridade materna em universitários. A categoria de insatisfeitos por excesso de peso foi significativamente associada à escolaridade materna, tanto na análise bruta quanto na ajustada. Essas estimativas 
26 | D.A.S. Silva, I.M.M. Pereira, A.C. Cabral de Oliveira

Tabela 1

Distribuição da amostra em relação às características sociodemográficas e perceção da imagem corporal $(n=217)$

\begin{tabular}{|c|c|c|c|}
\hline & $n$ & $\%$ & IC $(95 \%)$ \\
\hline \multicolumn{4}{|l|}{ Sexo } \\
\hline Feminino & 98 & 45.2 & $(38.5-51.8)$ \\
\hline Masculino & 119 & 54.8 & $(48.2-61.5)$ \\
\hline \multicolumn{4}{|l|}{ Idade } \\
\hline$\leq 20$ anos & 129 & 59.5 & $(52.9-66.0)$ \\
\hline$>20$ anos & 88 & 40.5 & $(34.0-47.1)$ \\
\hline \multicolumn{4}{|l|}{ Situação conjugal } \\
\hline Com companheiro & 16 & 7.4 & $(3.9-10.9)$ \\
\hline Sem companheiro & 201 & 92.6 & $(89.1-96.1)$ \\
\hline \multicolumn{4}{|l|}{ Nível econômico * } \\
\hline Classes A + B & 135 & 62.8 & $(56.3-69.3)$ \\
\hline Classes C + D & 80 & 37.2 & $(30.7-43.7)$ \\
\hline \multicolumn{4}{|l|}{ Curso } \\
\hline Licenciatura & 105 & 48.4 & $(41.7-55.1)$ \\
\hline Bacharelado & 112 & 51.6 & $(44.9-58.3)$ \\
\hline \multicolumn{4}{|l|}{ Escolaridade materna } \\
\hline$\leq 4$ anos & 26 & 12.0 & $(7.6-16.3)$ \\
\hline $5-8$ anos & 30 & 13.8 & $(9.2-18.5)$ \\
\hline $9-11$ anos & 89 & 41.0 & $(34.4-47.6)$ \\
\hline$\geq 12$ anos & 72 & 33.2 & $(26.9-39.5)$ \\
\hline \multicolumn{4}{|l|}{ Escolaridade paterna } \\
\hline$\leq 4$ anos & 23 & 10.6 & $(6.5-14.7)$ \\
\hline $5-8$ anos & 42 & 19.4 & $(14.1-24.7)$ \\
\hline $9-11$ anos & 79 & 36.4 & $(30.0-42.9)$ \\
\hline$\geq 12$ anos & 73 & 33.6 & $(27.3-40.0)$ \\
\hline \multicolumn{4}{|l|}{ Percepção da imagem corporal } \\
\hline Satisfeito & 67 & 30.9 & $(24.7-37.1)$ \\
\hline Insatisfeito por magreza & 89 & 41.0 & $(34.4-47.6)$ \\
\hline Insatisfeito por excesso de peso & 61 & 28.1 & $(22.1-34.1)$ \\
\hline
\end{tabular}

Nota: IC = intervalo de confiança; * variável com três respostas ignoradas

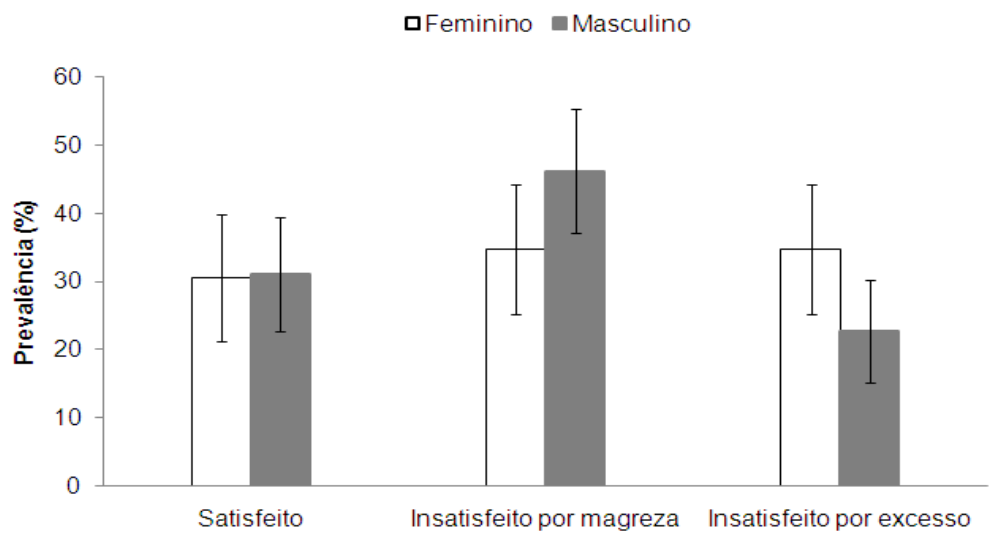

Figura 1. Prevalência de satisfação com a imagem corporal, insatisfação por magreza e insatisfação por excesso de peso acordo com o sexo 
indicaram que as chances de insatisfação por excesso de peso foram maiores nos acadêmicos com escolaridade materna superior a quatro anos (tendo como referência escolaridade materna $\leq 4$ anos), independentemente de sexo, idade, situação conjugal, nível econômico e curso. No entanto, não houve diferença significativa nas estimativas de odds ratio entre insatisfação por excesso de peso nos seguintes anos de escolaridade: $5-8,9-11$ e 12 ou mais anos de estudo, pois os intervalos de confiança se sobrepuseram.

Os resultados descritos na Tabela 3 indicaram que a imagem corporal não se associou à escolaridade paterna, tanto na análise bruta quanto na ajustada.

\section{DISCUSSÃO}

O presente estudo, que teve como objetivo verificar o impacto da escolaridade dos pais na perceção da imagem corporal em estudantes de Educação Física, apresentou, como principal achado, que a escolaridade materna, diferentemente da paterna, esteve associada à perceção da imagem corporal. Ademais, sujeitos cuja escolaridade materna foi superior a quatro anos apresentaram maior insatisfação por excesso de peso.

O nível de escolaridade dos pais é considerado indicador socioeconómico, que reflete diretamente na renda familiar. Estudos são consistentes em demonstrar que, quanto maior a escolaridade dos pais, maior é a disponibilidade de renda e de recursos materiais que a família tem à disposição (Lamy Filho et al., 2011). Nesse sentido, pode-se supor que os jovens do presente estudo, cujos pais têm maior grau de instrução, são os de maior renda familiar.

Torres, Marques, Ferreira e Bitar (2003), desenvolveram um estudo sobre segregação urbana no Brasil, com base nos dados do Censo Demográfico de 2000 e na utilização de Sistemas de Informação Geográfica, para o qual a cidade de São Paulo, maior metrópole da América Latina, serviu como escopo para as investigações. Os autores detetaram que o nível de escolaridade mais baixo dos pais significa uma capacidade reduzida de os filhos conseguirem um novo emprego ou renda em caso de desemprego, assim como piores ocupações quando estão empregados. Além disso, famílias chefiadas por pessoas com baixa escolaridade têm maior número de pessoas por domicílio, o que significa a necessidade de mais pessoas partilharem recursos comuns, trazendo consequências para a nutrição, a saúde e a educação. Por fim, renda mais baixa significa piores condições de nutrição e moradia, implicando uma probabilidade mais alta de doenças.

No Brasil, pessoas de baixa renda, em geral, residem em locais com condições precárias de educação, saúde, assistência social, esportes,

Tabela 2

Análise de regressão multinomial, odds ratio e intervalos de confiança entre imagem corporal (categoria de referência: satisfeito) e escolaridade materna

\begin{tabular}{|c|c|c|c|c|}
\hline & \multicolumn{2}{|c|}{ Insatisfeito por magreza } & \multicolumn{2}{|c|}{ Insatisfeito por excesso } \\
\hline & Bruta & Ajustada $^{a}$ & Bruta & Ajustada $^{a}$ \\
\hline & OR (IC 95\%) & OR (IC 95\%) & OR (IC 95\%) & OR (IC 95\%) \\
\hline \multicolumn{5}{|c|}{ Escolaridade materna } \\
\hline$\leq 4$ anos & 1.0 & 1.0 & 1.0 & 1.0 \\
\hline $5-8$ anos & $2.0(0.6-6.7)$ & $1.9(0.5-7.1)$ & $7.7(1.3-46.3)^{*}$ & $8.3(1.3-54.0)^{*}$ \\
\hline $9-11$ anos & $1.6(0.6-4.1)$ & $1.6(0.5-4.6)$ & $5.8(1.2-28.5)^{*}$ & $6.9(1.3-38.0)^{*}$ \\
\hline$\geq 12$ anos & $1.0(0.4-2.6)$ & $1.4(0.4-4.6)$ & $6.8(1.4-33.5)^{*}$ & $7.8(1.3-46.6)^{*}$ \\
\hline
\end{tabular}

Nota: OR = odds ratio, IC = intervalo de confiança; ${ }^{a}$ análise ajustada pelas variáveis: sexo, idade, situação conjugal, nível econômico e curso; * $p<.05$ 
Tabela 3

Análise de regressão multinomial, odds ratio e intervalos de confiança entre imagem corporal (categoria de referência: satisfeito) e escolaridade paterna

\begin{tabular}{|c|c|c|c|c|}
\hline & \multicolumn{2}{|c|}{ Insatisfeito por magreza } & \multicolumn{2}{|c|}{ Insatisfeito por excesso } \\
\hline & Bruta & Ajustada ${ }^{a}$ & Bruta & Ajustada ${ }^{a}$ \\
\hline & OR (IC 95\%) & OR (IC 95\%) & OR (IC 95\%) & OR (IC 95\%) \\
\hline \multicolumn{5}{|c|}{ Escolaridade paterna } \\
\hline$\leq 4$ anos & 1.0 & 1.0 & 1.0 & 1.0 \\
\hline $5-8$ anos & $0.7(0.2-2.5)$ & $0.6(0.2-2.2)$ & $2.7(0.6-11.5)$ & $2.7(0.6-12.2)$ \\
\hline $9-11$ anos & $0.8(0.3-2.3)$ & $0.8(0.2-2.4)$ & $1.3(0.3-5.2)$ & $1.1(0.3-4.9)$ \\
\hline$\geq 12$ anos & $0.7(0.3-2.0)$ & $0.7(0.2-2.5)$ & $1.5(0.4-5.9)$ & $1.2(0.3-5.5)$ \\
\hline
\end{tabular}

Nota: OR = odds ratio, IC = intervalo de confiança; ${ }^{a}$ análise ajustada pelas variáveis: sexo, idade, situação conjugal, nível econômico e curso

cultura e lazer (Torres et al., 2003). Esse fato reflete nas relações sociais, que se tornam reduzidas, e no acesso escasso aos meios de comunicação durante a infância e a adolescência, períodos em que, geralmente, a perceção do corpo é construída (Dohnt \& Tiggemann, 2006; Pelican et al., 2005).

Pelican et al. (2005) realizaram um estudo com 103 adultos, cujo objetivo era verificar o poder que as relações sociais exerciam na perceção da imagem corporal. Os autores evidenciaram que alguns sujeitos se sentiam "envergonhados" por causa de comentários que outras pessoas teciam em relação ao seu corpo e esse fato refletia na insatisfação com a própria imagem corporal. Dohnt e Tiggemann (2006) investigaram a influência dos pares e da mídia na perceção da imagem corporal em meninas de cinco a oito anos de idade. Constataram que a imagem que os pares têm de seu corpo induz a imagem que as meninas têm de si mesmas. Além disso, os autores detetaram que a televisão e as revistas funcionam como meios de se aprenderem técnicas de emagrecimento, assim como são meios que resultam em maior insatisfação com a imagem corporal, devido às pessoas que aparecem nesses meios de comunicação apresentarem corpos belos que, muitas vezes, são esculpidos por meio de cirurgias plásticas.

$\mathrm{O}$ presente estudo evidenciou que, independentemente de sexo, idade, situação conjugal, nível econômico e curso do estudante, a insatisfação por excesso de peso foi em torno de sete a oito vezes maior nos acadêmicos com escolaridade materna superior a quatro anos. A escolaridade materna está associada a comportamentos relacionados à saúde e tem impacto sobre o desenvolvimento de crianças e adolescentes por meio de fatores como organização do ambiente, expectativas e práticas parentais (Andrade, Santos, Bastos, Pedromonico, Almeida-Filho, \& Barreto, 2005). Além disso, alto nível de escolaridade materna é apontado como fator de proteção contra mortalidade infantil, baixo peso ao nascer e hábitos inadequados de estilo de vida, pois influencia no desenvolvimento saudável do jovem, na extensão de vocabulário, nos escores de inteligência e no estilo de vida saudável (Andrade et al., 2005; Haidar, Oliveira, \& Nascimento, 2001).

Nenhuma associação foi encontrada entre perceção da imagem corporal e nível de escolaridade paterna no presente estudo. O nível de escolaridade paterna tem bastante relevância na aquisição da renda familiar (Carvalho \& Almeida, 2003). No entanto, a literatura não reporta associações consistentes entre escolaridade paterna e comportamentos relacionados à saúde dos filhos. Estudos descrevem que o papel da mãe tem influencia direta na criação dos filhos e no status de saúde da criança e do adolescente (Carvalho \& 
Almeida, 2003; Haidar, Oliveira, \& Nascimento, 2001). Em muitas situações, os pais são responsáveis por trabalhar fora de casa e trazer o sustento da família, enquanto as mães ficam no lar e cuidam da casa e da criação dos filhos, que acabam por refletir costumes e modos de pensar e se relacionar da mãe (Carvalho \& Almeida, 2003). Por essas razões, acredita-se que o presente estudo encontrou associação entre perceção da imagem corporal e nível de escolaridade materna.

A presente investigação encontrou prevalência de insatisfação com a imagem corporal entre $69.1 \%$ dos acadêmicos de Educação Física. Estudo desenvolvido em amostra representativa de estudantes de diversos cursos de uma universidade do Sul do Brasil encontrou prevalência de insatisfação com a imagem corporal de $77.6 \%$ (Quadros et al., 2010). Em pesquisa desenvolvida com académicos do curso de Nutrição da cidade do Rio de Janeiro, Brasil, foi verificado insatisfação com a imagem corporal em $49.4 \%$ dos alunos (Bosi, Luiz, Morgado, Costa, \& Carvalho, 2006). Em relação a pesquisas desenvolvidas, exclusivamente, com acadêmicos de Educação Física, Rech, Araújo e Vanat (2010) desenvolveram um estudo transversal com 294 estudantes da cidade de Ponta Grossa, Paraná, Brasil e encontraram que a prevalência de insatisfação com a imagem corporal foi de $61.2 \%$. Sousa (2010) investigou estudantes de Educação Física na região Nordeste do Brasil e detetou que dos 105 sujeitos analisados, $63.8 \%$ estavam insatisfeitos com a aparência física.

Esses dados revelam que mesmo entre estudantes de Educação Física, que durante a formação universitária têm disciplinas que tratam diretamente do corpo e da relação com o mundo, a insatisfação com a imagem corporal é elevada. Esse achado preocupa, pois como é colocado por O'Dea e Abraham (2001), muitos estudantes de Educação Física se tornarão professores de crianças e adolescentes e serão responsáveis por falar da relação do corpo humano com a sociedade e da importância de hábitos saudáveis para a saúde, bem como tratarão diretamente da manifestação corporal com os jovens. Assim, à medida que estudantes de Educação Física reportam insatisfação com a imagem corporal durante a formação, isso pode refletir nos conteúdos que serão passados futuramente para os jovens e torná-los, também, insatisfeitos com o corpo, resultando em adoção de hábitos inadequados para a busca do ideal de beleza.

Pesquisas com universitários de diversos cursos (Quadros et al, 2010) e com acadêmicos de Educação Física (Rech, Araújo, \& Vanat, 2010; Sousa, 2010) reportaram que as mulheres estavam mais insatisfeitas por excesso de peso, enquanto os homens eram insatisfeitos por magreza. O presente estudo encontrou uma tendência semelhante entre os universitários investigados; todavia, tais valores não apresentaram diferenças significantes estatisticamente entre homens e mulheres em relação ao tipo de insatisfação com a imagem corporal, pois os intervalos de confiança entre as proporções se sobrepõem (Figura 1). Isso revela que entre acadêmicos do atual estudo o tipo de insatisfação com a imagem corporal é semelhante de acordo com o sexo e que iniciativas de conscientização com o corpo por parte da instituição investigada podem ser tomadas para o combate da insatisfação por magreza ou por excesso de peso independente se os alunos são homens ou mulheres.

O presente estudo apresenta como limitações o desenho transversal, que não permite identificar relações de causalidade entre a perceção da imagem corporal e a escolaridade dos pais. Além disso, a utilização de desenhos bidimensionais das silhuetas corporais para a avaliação da imagem corporal pode implicar falhas na representação total do corpo e/ou na distribuição da massa de gordura. Contudo, cabe ressaltar que esse trabalho foi o primeiro estudo sobre perceção da imagem corporal que investigou a associação com a escolaridade dos pais e pode servir como informação útil para políticas públicas de incentivo à educação formal e à conscientização corporal. 
Conclui-se que, independentemente de sexo, idade, situação conjugal, nível econômico e curso dos estudantes, a perceção da imagem corporal esteve associada à escolaridade materna, sobretudo em acadêmicos insatisfeitos por excesso de peso. Além disso, universitários cujas mães estudaram mais de quatro anos são mais insatisfeitos por excesso de peso em comparação àqueles que as mães têm até quatro anos de estudo. Esse fato se agrega a outros, que reportam a necessidade de uma educação básica e superior de qualidade, que trate de assuntos vinculados à perceção corporal e à adoção de hábitos saudáveis, o que resultará em perceção positiva da imagem corporal por parte dos pais e dos filhos.

\section{Agradecimentos:}

Os autores gostariam de agradecer ao Departamento de Educação Física do Centro de Ciências Biológicas e da Saúde da Universidade Federal de Sergipe pelo apoio durante a coleta de dados.

\section{Conflito de Interesses:}

Nada a declarar.

\section{Financiamento:}

Nada a declarar.

\section{REFERÊNCIAS}

Abraham, S. F. (2003). Dieting, body weight, body image and self-esteem in young women: doctors' dilemmas. The Medical Journal of Australia, 178(12), 607-611.

Al Sabbah, H., Verercken, C., Elgar, F., Nansel, T., Aasvee, K., Abdeen, Z., Ojala, K., Ahluwalia, N., \& Mes, L. (2009). Body weight dissatisfaction and communication with parents among adolescents in 24 countries: International cross-sectional survey. BMC Public Health, 9, 52. doi: 10.1186/1471-2458-9-52

Alves, D., Pinto, M., Alves, S., Mota, A., \& Leirós, V. (2009). Cultura e imagem corporal. Motricidade, 5(1), 1-20.

Andrade, A., Santos, D., Bastos, A., Pedromonico, M., Almeida-Filho, N., \& Barreto, M. (2005). Ambiente familiar e desenvolvimento cognitivo infantil: Uma abordagem epidemiológica. Re- vista de Saúde Pública, 39(4), 606-611. doi: 10.15 90/S0034-89102005000400014

Associação Brasileira de Empresas de Pesquisa ABEP (2008). Critério Padrão de Classificação Econômica Brasil/2008. Consultado em 26 de agosto de 2009, a partir de www.abep.org/ codigosguias/Criterio_Brasil_2008.pdf

Bosi, M. L., Luiz, R. R., Morgado, C. M., Costa, M. L., \& Carvalho, R. J. (2006). Autopercepção da imagem corporal entre estudantes de nutrição: Um estudo no município do Rio de Janeiro. Jornal Brasileiro de Psiquiatria, 55(2), 108-113.

Cafri, G., \& Thompson, J. K. (2004). Evaluating the convergence of muscle appearance attitude measures. Assessment, 11(3), 224-229. doi: 10.1 177/1073191104267652

Carvalho, I. M., \& Almeida, P. H. (2003). Família e proteção social. São Paulo em Perspectiva, 17(2), 109-122.

Dohnt, H., \& Tiggemann, M. (2006). Body image concerns in young girls: The role of peers and media prior to adolescence. Journal of Youth and Adolescence, 34, 1-11. doi: 10.1007/s10964-0059020-7

Frost, L. (2005). Theorizing the young woman in the body. Body and Society, 11(1), 63-85. doi: 10. 1177/1357034X05049851

Haidar, F. H, Oliveira, U. F., \& Nascimento, L. F. (2001). Escolaridade materna: Correlação com os indicadores obstétricos. Cadernos de Saúde Publica, 17(4), 1025-1029. doi: 10.1590/S0102311X2001000400037

Kappel, D. B. (2007). Índice de desenvolvimento infantil no Brasil: Uma análise regional. Revista Brasileira de Educação, 12(35), 232-240. doi: 10.1 590/S1413-24782007000200004

Lamy Filho, F., Medeiros, S. M., Lamy, Z. C., \& Moreira, M. E. (2011). Ambiente domiciliar e alterações do desenvolvimento em crianças de comunidade da periferia de São Luís - MA. Ciência E Saúde Coletiva, 16(10), 4181-4187. doi: 10.1590/S1413-81232011001100023

Muennig, P., Jia, H., Lee, R., \& Lubetkin, E. (2008). I think therefore I am: Perceived ideal weight as a determinant of health. American Journal of Public Health, 98(3), 501-506.

Niskar, A., Baron-Epel, O., Gartysandalon, N., \& Keinan-Boker, L. (2009). Body weight dissatisfaction among Israeli Jewish and Arab women with normal or overweight-obese body mass index, Israeli INHIS-1, 2003-2004. Preventing Chronic Disease, 6(2), A51. 
O'Dea, J. A., \& Abraham, S. (2001). Knowledge, beliefs, attitudes, and behaviors related to weight control, eating disorders, and body image in Australian trainee home economics and physical education teachers. Journal of Nutrition Education, 33(6), 332-340.

Pelican, S., Heede, F., Holmes, B., Melcher, L., Wardlaw, H., Raidl, M., ... Moore, S. (2005). The power of others to shape our identity: Body image, physical abilities and body weight. Family and Consumer Sciences Research Journal, 34(1), 56-79. doi: 10.1177/1077727X0527773

Pimenta, A. M., Sánchez-Villegas, A., Bes-Rastrollo, M., López, C. N, \& Martínez-González, M. A. (2009). Relationship between body image disturbance and incidence of depression: The SUN prospective cohort. BMC Public Health, 9, 1. doi: 10.1186/1471-2458-9-1

Quadros, T. M., Gordia A. P., Martins C. R., Silva, D. A., Ferrari E. P., \& Petroski, E. L. (2010). Imagem corporal em universitários: Associação com estado nutricional e sexo. Motriz, 16(1), 78-85. doi: 10.5016/1980-6574.2010v16n1p78

Rech, C., Araújo, E., \& Vanat, J. (2010). Autopercepção da imagem corporal em estudantes do curso de educação física. Revista Brasileira de Educação Física e Esporte, 24(2), 285-292.

Slade, P. D. (1994). What is body image? Behaviour Research and Therapy, 32 (5), 325-331.

Sousa, T. F. (2010). Insatisfação com o peso corporal em universitários do curso de Educação Física no Nordeste do Brasil. Lecturas Educación Física y Deportes, 14(142).

Stunkard, A., Sorensen, T., \& Schulsinger, F. (1983). Use of the Danish adoption register for the study of obesity and thinness. In S. Kety, L. Roland, R. Sidman, \& S. Matthysse (Eds.), The genetics of neurological and psychiatric disorders (pp. 115-120). New York, NY: Raven Press.

Torres, H., Marques, E., Ferreira, M., \& Bitar, S. (2003). Pobreza e espaço: Padrões de segregação em São Paulo. Estudos Avançados, 17(47), 97-128.

Yeh, H., Tzeng, N.., Chu, H., Chou, Y. H., Lu, R. B., O'Brien, A., ... Chou, K. R. (2009). The risk of eating disorders among female under-graduates in Taiwan. Archives of Psychiatric Nursing, 23(6), 430-440. doi: 10.1016/j.apnu.2008.12.001

(c) EY-Ne Todo o conteúdo da revista Motricidade está licenciado sob a Creative Commons, exceto quando especificado em contrário e nos conteúdos retirados de outras fontes bibliográficas. 